\title{
Multiobjective Optimal Design of Underwater Acoustic Projector with Porous Piezocomposite Active Elements
}

\author{
A. V. Nasedkin ${ }^{1}$, M. S. Shevtsova ${ }^{1}$, J.-C. Liu ${ }^{2}$, S.-H. Chang ${ }^{3}$, J.-K. Wu ${ }^{3}$ \\ ${ }^{1}$ Mathematical Modeling Department, Southern Federal University, Rostov-on-Don, Russia \\ ${ }^{2}$ Department of Electrical Engineering, National Taiwan Ocean University, Zhongzheng, Taiwan \\ ${ }^{3}$ Departments of Microelectronics \& Marine Engineering, National Kaoshiung Marine University, Kaoshiung, Taiwan \\ Email: mariamarcs@bk.ru
}

Received October 2013

\begin{abstract}
This paper concerns the optimization problem for multilayered ultrasonic transducer with active porous piezoelectric layer. The dependences of the effective moduli for porous piezoelectric material on porosity have been previously obtained and allowed to decrease the number of design variables. The multiobjective optimization problem based on the Pareto-frontier calculation has been solved using the live-link of finite-element (FE) package Comsol Multiphysics with MATLAB.
\end{abstract}

Keywords: Porous Piezoceramics; Multiobjective Optimization; Pareto Frontier; Multilayered Acoustic Projector; Underwater Applications

\section{Introduction}

Porous piezoceramic materials are widely used in medical imaging, ultrasonic transducers, hydrophones and other piezoelectric devices. A large number of investigations are referred to the development of effective structures for acoustic transducers based on the porous piezoelectric materials due to their high piezoelectric sensitivity, extended frequency bandwidth and better matching to the acoustic medium. The aim of presented work is the developing of methods for synthesizing the optimal structures of the transducers for underwater applications. On the base of developed numerical method for the porous materials effective moduli determine the optimization problem statement and solving for whole device structure have been implemented. Hydrophones, projectors and combined devices - transceivers are the general types of modern devices used for sonar and underwater transmission. A projector is a transducer used for generating sound and transmitting it to the acoustic medium. In order to effectively fulfill its purpose, underwater acoustic transducers have to meet many requirements, including: low impedance for better acoustic matching with water, durability; high sensitivity; ability to take high mechanical load and hydrostatic pressure. The most widely used devices in underwater acoustics are the devices based on the piezoelectric effect. The expansion of their effective applications caused by both the success of the piezoelectric materials science and the developing of the micro-electromechanical systems (MEMS), technologies to create devices based on nanoscale multilayer active hydrophones and projectors, arrays of transducers for receiving and radiation of directed sound [1]. One of the problems at realizing such structures is the impedance matching. The use of intermediate layers allows to overcoming this difficulty. However in case of active element made of dense piezoelectric ceramics a number of layers are needed; and this involves big energy losses of the whole structure. The effective method for getting over this difficulty is the use of porous piezoelectric ceramics. This entails both decreased number of intermediate layers and better acoustic agreement between the transducer and medium. Various experimental [2,3] and theoretical $[4,5]$ investigations show, that porous piezoelectric ceramics may significantly improve the desired properties of transducers and expand the use of piezoelectric materials. Porous piezocomposite materials are characterized by lower values of the transverse piezoelectric modulus $d_{31}$ at almost the same values of the thickness piezoelectric modulus $d_{33}$ [6,7] responsible for the efficient conversion of electrical and mechanical energy. Therefore, the use of porous materials in piezoelectric ultrasonic transducers, hydrophone structures and other piezoelectric devices is very promising today.

In previous works $[6,7]$ it was investigated how the properties of piezoelectric materials depend on porosity 
for the ceramics of different connectivity and ferroelectric hardness. Such dependencies have been obtained for the full set of material constants.

The aim of presented research is a structural optimization of multilayered transducer with the porous piezoelectric active element which all material's parameter are expressed through the value of porosity. The previously obtained dependencies of effective moduli allow to sufficiently decrease the number of design variables. For the underwater acoustic projector which structure contains such layers as: an acoustic window, matching, piezoelectric, backing plate and protective foam layers (see Figure 1) we formulate the coupled problem of acoustics and electroelasticity in axial-symmetric formulation. As the optimized objectives we introduce averaged sound pressure level (SPL), transmitting current response (TCR) and the mean-square value of the SPL irregularity in frequency range from 100 to $400 \mathrm{kHz}$. The design variables are: Young's modules of an acoustic window layer, protective foam layer, and matching layer; mass damping parameter and stiffness damping parameter of layers; porosity of an active piezoelectric layer. In this investigation we use the approach based on the Pareto-frontier calculation, i.e. building the set of points in the $6 \mathrm{D}$ space of design variables where all the objectives are feasible.

The coupled problem is numerically implemented by the live-link of the FE package Comsol Multiphysics with MATLAB. The best results have been obtained at about $30 \%-40 \%$ of porosity for piezoelectric layer and

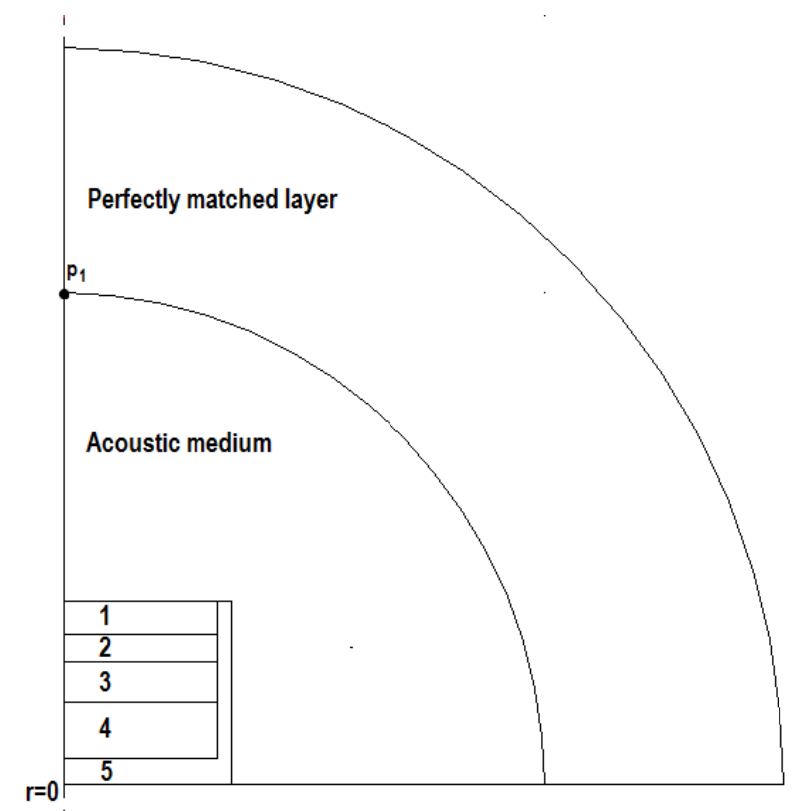

Figure 1. The scheme of multilayered transducer placed in acoustic medium: 1-acoustic window layer, 2-matching layer, 3-active porous piezoelectric layer, 4-backing plate, 5-protective foam layer. mechanical and damping parameters of intermediate layers that may characterize a wide range of polymeric materials. Electro-acoustic efficiency, defined by the TCR parameter, is significantly higher for porous material than for the dense ceramics. Obtained data and proposed methods might be effectively used for the structural optimization of enough wide range of transducers.

\section{Dependencies of the Effective Moduli on Porosity}

For the effective properties determination we used an approach presented in details in $[6,7]$ and based on the effective moduli methods [2,6-8], modeling of representative volumes for porous piezoelectric materials and the use of the FE technologies. There are several methods to obtain the structure of a two-phase cubic piezoelectric composite material. At low percent of porosity a simple but an adequate microstructure of porous material is a model of the piezoceramic cubic lattice consisting of unit cells - cubes, some of which are randomly declared to be pores [2]. However, this model may lose connectivity of a skeleton at a large number of pores. In order to overcome this problem the algorithms based on the percolation theory can be used. In case of low porosity the clusters can be built from pores, while at high porosityfrom piezoelectric material. One of such methods is Witten-Sander's method [9] has been analyzed in [8]. In presented work both methods: random method and Witten-Sander's method were used.

The experimental data [2,4,10-12] were compared with the obtained results for a porous material with medium ferroelectric hardness PZT-4. For all the figures, presented below, the curves, related to the random method, marked by circles, and the Witten-Sander method, by triangles. The dependences, obtained without taking into account the inhomogeneity of the polarization field, correspond to dotted lines; dash-dot lines correspond to calculations performed at taking into account the nonuniform polarization; and the dashed lines indicate the experimental data.

Figure 2 shows the relative electric permittivity $r\left(\varepsilon_{33}^{S}\right)=\varepsilon_{33}^{S}(p) / \varepsilon_{33}^{S}(0)$ on porosity $p$ in comparison with the experimental data $[10,11]$. One can conclude that the dependencies are linear for both random and WittenSander methods. The results obtained with the hypothesis of polarization field's inhomogeneity differ from those obtained without this hypothesis less than $1 \%-2 \%$. It also can be seen that all four dependencies are very close, and numerical results are in better agreement with the data from [10]. The values of electric permittivity obtained in [11] strongly decrease with increasing porosity and give about $35 \%$ discrepancy with the calculated data and the results from [9]. 


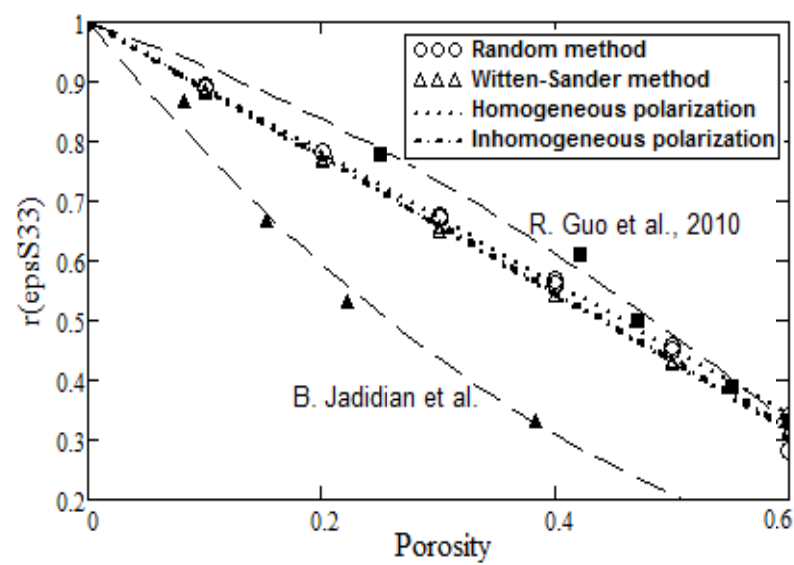

Figure 2. The dependency of relative electric permittivity on porosity.

After the calculation the full set of the effective moduli $c_{\alpha \beta}^{E}(p), e_{i \alpha}(p), \varepsilon_{i i}^{S}(p)$, we obtained such important characteristics of piezoelectric ceramics as piezoelectric coefficients $d_{31}$ and $d_{33} \quad\left(d_{i \alpha}=d_{i \alpha}(p)=e_{i \beta}(p) s_{\alpha \beta}^{E}(p)\right.$, where $s_{\alpha \beta}^{\text {Eeff }}$ are the components of the compliance matrix). The comparison of the obtained dependencies with the experimental data $[2,4,13]$ are performed for both longitudinal (Figure 3(a)) and transversal (Figure 3(b)) piezoelectric moduli.

As can be seen the dependency for $d_{31}$ coefficient, related to the Witten-Sander method, significantly decreases with increasing porosity compared to the random method. It is obvious that the relationship obtained at the assuming of polarization field's inhomogeneity is in a better agreement with the experimental data (Figure 3(a)). At the same time, the dependencies for the piezoelectric modulus $d_{33}$ are essentially independent of porosity for both methods (Figure 3(b)). It should be noted that the maximum error is still quite significant and is approximately $10 \%-15 \%$ for the data from [13], and about $3 \%$ - $5 \%$ for the data $[2,4]$.

At the second stage of the presented investigation, during the simulation of a multilayer transducer's structure, the obtained dependencies at the assuming of the polarization field's inhomogeneity and related to the Witten-Sander method have been accepted as material properties for the active element.

\section{Coupled Problem of Acoustics and Electroelasticity for a Multilayered Piezoelectric Transducer}

In presented investigation, we consider a model of an ultrasound piezoelectric transducer for underwater applications in its axial-symmetric formulation. Cylindrical surface and the bottom of a transducer are covered with a protective layer made of foam. Transducer consists of
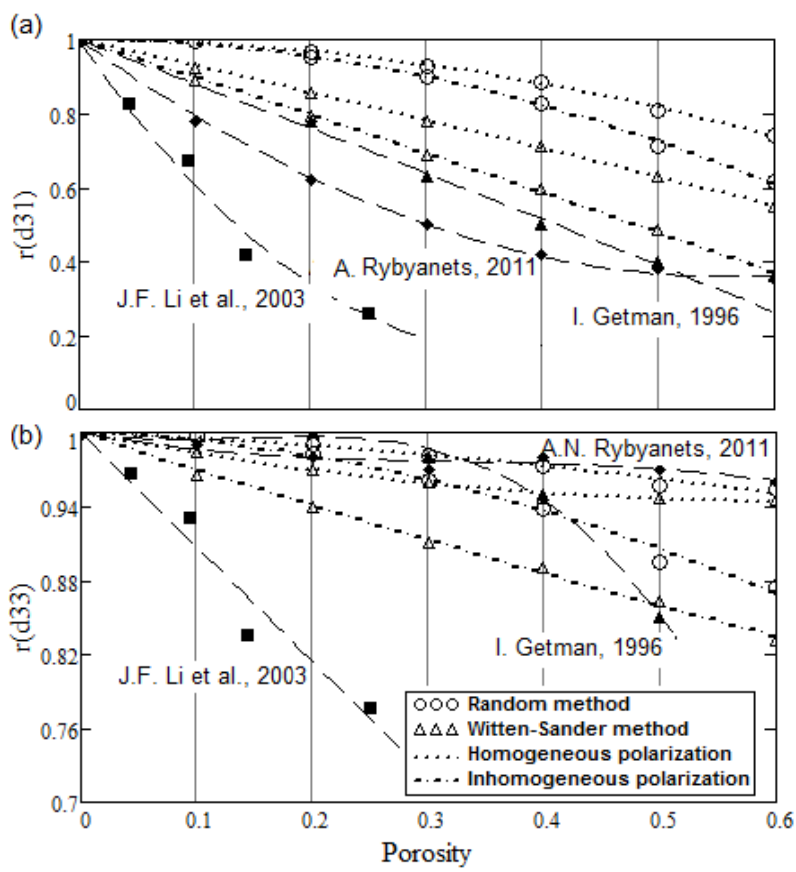

Figure 3. The dependencies of relative piezoelectric coefficients on porosity.

four layers, such as: backing plate, piezoelectric active layer, matching layer and acoustic window. The whole construction is surrounded with an acoustic medium. On the boundary of an acoustic medium the perfectly matched layer (PML) is placed so that the waves, incident upon the PML from a non-PML medium, do not reflect at the interface (Figure 1). The thicknesses of the piezoelectric layer, protective and backing plate layers have been chosen to be approximately a half length of the longitudinal wave at thickness vibration mode; an acoustic window thickness-approximately $3 / 4$, and matching layer-1/4 of the longitudinal waves at the same frequency of about $300 \mathrm{kHz}$. We formulate the coupled problem of acoustics and electroelasticity using the FE package COMSOL Multiphysics and its two application modes: Pressure Acoustics mode (time-harmonic analysis) and Piezo Axial Symmetry mode (frequency response analysis). Sound waves are governed by the inhomogeneous Helmholtz equation for the acoustic pressure $p$ :

$$
\nabla \cdot\left(-\frac{1}{\rho_{0}} \nabla p\right)-\frac{\omega^{2} p}{\rho_{0} c_{s}^{2}}=0,
$$

where $\rho_{0}=1000 \mathrm{~kg} / \mathrm{m}^{3}$ is a fluid density, $c_{s}=1500 \mathrm{~m} / \mathrm{s}$ is a speed of sound, $\omega=2 \pi f(\mathrm{rad} / \mathrm{s})$ is the angular frequency with $f(\mathrm{~Hz})$ denoting the frequency. The boundary conditions are following: sound hard wall (the boundary between an acoustic medium and the PML) and axial symmetry (on the left boundary), (see Figure 1). 
The constitutive relations for the piezoelectric active layer are taken in the stress-charge form as follows:

$$
\begin{gathered}
\boldsymbol{\sigma}=\mathbf{c}^{E} \cdot \boldsymbol{\varepsilon}-\mathbf{e}^{*} \cdot \mathbf{E}, \\
\mathbf{D}=\mathbf{e} \cdot \boldsymbol{\varepsilon}+\boldsymbol{\varepsilon}^{S} \cdot \mathbf{E}
\end{gathered}
$$

where $\boldsymbol{\varepsilon}$ is the strain tensor, $\boldsymbol{\sigma}$ is the stress tensor, $\mathbf{E}$ is the electric field vector, $\mathbf{D}$ is the electric displacement vector; $\mathbf{c}^{E}$ is the tensor of elastic stiffness moduli at constant electric field; e is the tensor of piezoelectric moduli (stress coefficients); $\boldsymbol{\varepsilon}^{S}$ is the tensor of electric permittivity moduli at constant mechanical stress.

Coupling between solid end acoustic media is provided by the boundary conditions: the top and the sides of a transducer undergo both an acoustic pressure and the inward accelerations. The bottom of a transducer is fixed; on the left boundary we consider an axial symmetry condition; on the top of piezoelectric layer the constant electric potential with amplitude $100 \mathrm{~V}$ in whole studied frequency band is applied, when the bottom is grounded.

Since the dimensions of the investigated transducer are quite small, this type of projector cannot be used to generate directional sound and therefore we will consider the sound pressure level only in a direct ray. When the transducer is placed into acoustic medium the thickness vibration mode is excited at frequencies from approximately 100 to $400 \mathrm{kHz}$. This frequency range was used during the following optimization of transducer's parameters.

\section{Multiobjective Optimization of the Piezoelectric Transducer}

There is a wide range of materials that can be used as the constituent layers of a transducer; this proves the possibility to vary their mechanical properties within the wide scope. It should be noted that we chose tungsten as a backing plate material to generate the thickness vibration mode of a PZT layer because of its large mechanical stiffness and high acoustic impedance. In order to formulate the optimization problem let us introduce six design variables: porosity of an active layer (por), Young's modules of an acoustic window layer $\left(E_{a w}\right)$, matching layer $\left(E_{m}\right)$, and protective foam layer $\left(E_{f}\right)$; mass damping parameter $\left(R_{1}\right)$ and stiffness damping parameter $\left(R_{2}\right)$ of layers. In our investigation we considered three objectives: sound pressure level (SPL) in direct ray measured at the $1 \mathrm{~m}$ distance from the sound source and transmitting current response (TCR) to be maximized; the deviation of SPL is to be minimized. SPL is represented in decibels as follows

$$
p=20 \lg \left(\left|p_{1}\right| / p_{\text {ref }}\right),
$$

where $p_{1}$ is the sound pressure at the measurement point; and $p_{\text {ref }}=2 \times 10^{-5} \mathrm{~Pa}$ is the threshold of sound pressure. TCR is the ratio of an absolute value of sound pressure $p_{1}$, to the amplitude of electric current $I$ through the active element:

$$
S_{I}=\left|p_{1}\right| / I \text {. }
$$

There is a wide range of approaches to structural optimization. In the framework of multi-criteria optimization problem (MOO) when several objective functions exist, there is no unique solution, but a number of optimum solutions exist. In this situation the most suitable way to optimization is a calculation of a so-called Pareto optimum or Pareto-frontier. Using the Pareto approach we suppose the assignment of a set of choices for all objective components that are Pareto efficient. By confining the set of choices to only the Pareto-efficients instead of considering the full range for each parameter, it is possible to make trade-offs within this set. During the solving of considered optimization problem the three integrals were assumed to be optimized:

$$
\begin{gathered}
\langle p\rangle=\int_{f_{1}}^{f_{2}} p(f) d f /\left(f_{2}-f_{1}\right), \\
\langle\Delta p\rangle=\sqrt{\frac{\int_{f_{1}}^{f_{2}}|\langle p\rangle-p(f)|^{2} d f}{f_{2}-f_{1}}}, \\
\langle T C R\rangle=\int_{f_{1}}^{f_{2}} \frac{p(f)}{I(f)} d f /\left(f_{2}-f_{1}\right),
\end{gathered}
$$

where $\langle p\rangle,\langle\Delta p\rangle$ and $\langle T C R\rangle$ represent an averaged SPL, deviation of SPL and TCR, respectively; $f_{1}$ and $f_{2}$ are the boundaries of the frequency range.

Obviously the construction of a Pareto-frontier was complicated for the three-dimensional space of objective functions. In order to overcome this difficulty the illustration of a Pareto-frontier has been represented using the level lines. At the numerical problem solving MATLAB varies design parameters for the transducer, calls the FE model simulated by Comsol Multiphysics, and is carry out the multiple computations of the objectives. Then obtained data are being analyzed and illustrated using the set of complimentary procedures, written in MATLAB (see Figures 4(a), (b)).

\section{Numerical Results and Discussion}

At the analysis of the simulation results it was founded that the influence of both damping designs variables on the objectives are negligible. So only two obtained projections of the criteria points set on the spaces of other design variables are presented in Figure 4. Figure 4(a) corresponds to the projection on subspace of two design variables: porosity of active layer and Young's modulus 


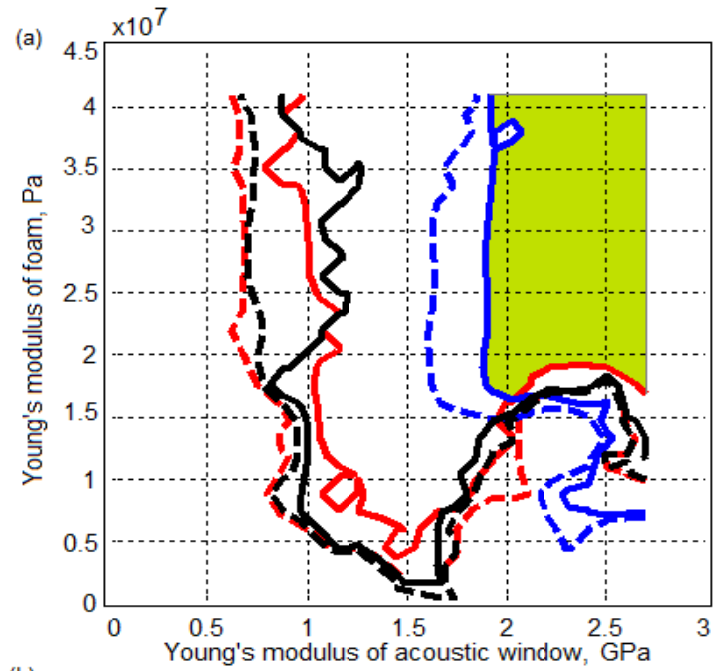

(b)

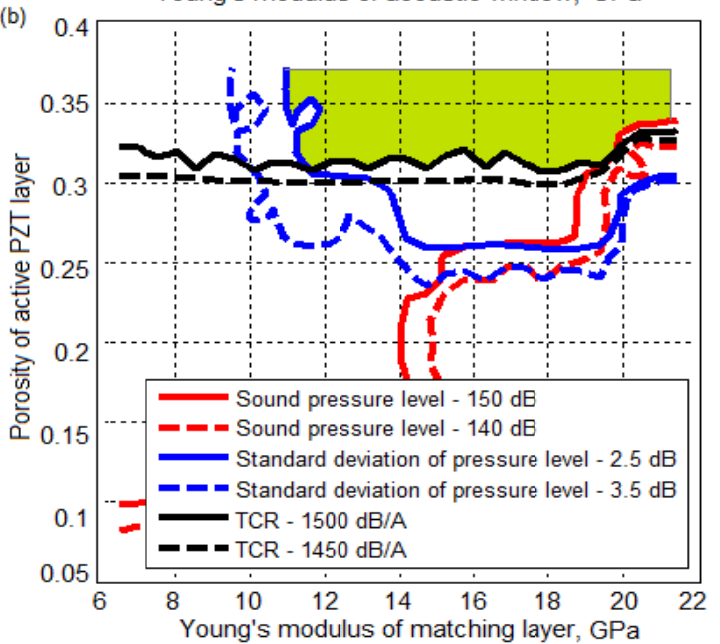

Figure 4. Projections of the criteria set presented as the contour lines of objectives levels on the subspaces of design variables.

of matching layer; Figure 4(b) for Young's modulus of side protective layer and Young's modulus of acoustic window. For the studied objectives we used the following bounds for the feasible values: $\geq 150 \mathrm{~dB}$ for the SPL, $\geq 1500 \mathrm{~dB} / \mathrm{A}-$ for TCR, and $\leq 2.5 \mathrm{~dB}$ for the deviation of SPL. On the presented figures the areas between solid and dashed lines are the projections of Pareto frontier on the 2D subspaces of the design variables. Green areas represent the intersections of optimum areas. It can be seen from Figure 4(a) that the energetic efficiency, characterized by TCR, reaches desirable values when the porosity of active layer is greater than 0.3 . However, the maximum values of SPL are reached at a considerable variation of porosity. One can observe that the optimum SPL value shifts with the growth of Young's modulus of matching layer from about 1.6 GPa till 2.2 GPa at higher porosity; on the other hand the lower Young's modulus corresponds to lower porosity. The most uniform frequency response of SPL are reached when the percent of porosity is greater than 0.25. It's obvious (Figure 4(b)) that the Young's modulus of acoustic window significantly influences on the uniformity of the SPL's frequency response; the optimum quantities correspond to the values greater than or equal to $2 \mathrm{GPa}$. At the same time the Young's moduli of a protective and acoustic window layers does not impact on TCR in optimum areas.

For clarity, we present below three groups of frequency responses obtained for the three sets of values of the design variables that is contained in Table $\mathbf{1}$.

For the first set all the design variables were taken from the obtained optimum areas. The second set contains parameters being outside the Pareto frontier. The last set corresponds to a transducer with an active layer made of dense piezoelectric ceramics; the first parameter (Young's modulus of acoustic window) was taken outside the optimum area, the other four variables belong to the Pareto frontier.

For each set of design variables the frequency responses for SPL and TCR are calculated and plotted on the Figures 5(a) and 5(b), respectively.

The graphs shown in Figure 5, a clearly shows that the optimal set of design parameters provides a much less unevenness of sound pressure level as comparing to the cases of active element with dense ceramics (3) and 20\% porosity. The maximum deviation of the sound pressure level in the investigated frequency range for the designs (1), (2), and (3) are $8.5 \mathrm{~dB} / 21 \mathrm{~dB} / 12 \mathrm{~dB}$, respectively. The sound pressure level inside the whole frequency range is the best. It exceeds the SPL of projector on the base of dense ceramics on 5\%, and SPL for design (2)on $10 \%$. The peak observed in the TCR graph (see Figure 5(b)) shows that a transducer (1) demonstrates the utmost resonance properties when the design variables are taken from the optimal area. If the electronic exciting device has a sufficient performance that allows to maintaining a constant amplitude of the applied potential in the whole frequency range, the resonance features of TCR does not worsen the uniformity of the frequency response for the sound pressure.

\section{Conclusion}

Ten dependencies of material constants on porosity were

Table 1. Design variables for the three examples of simulated projectors.

\begin{tabular}{ccccccc}
\hline $\begin{array}{c}\text { Studied } \\
\text { design }\end{array}$ & $\begin{array}{c}E_{a w}, \\
\mathrm{GPa}\end{array}$ & $\begin{array}{c}E_{f}, \\
\mathrm{MPa}\end{array}$ & $\begin{array}{c}E_{m}, \\
\mathrm{GPa}\end{array}$ & $\begin{array}{c}R_{1} \\
\times 10^{-7}\end{array}$ & $\begin{array}{c}R_{2} \\
\times 10^{-7}\end{array}$ & por \\
\hline $\begin{array}{c}\text { Inside the } \\
\text { optimum area }\end{array}$ & 2.5 & 30 & 16 & 1.5 & 0.6 & 0.4 \\
$\begin{array}{c}\text { Outside the } \\
\text { optimum area } \\
\text { Dense PZT }\end{array}$ & 0.5 & 5 & 20 & 2.3 & 1.2 & 0.2 \\
\hline
\end{tabular}




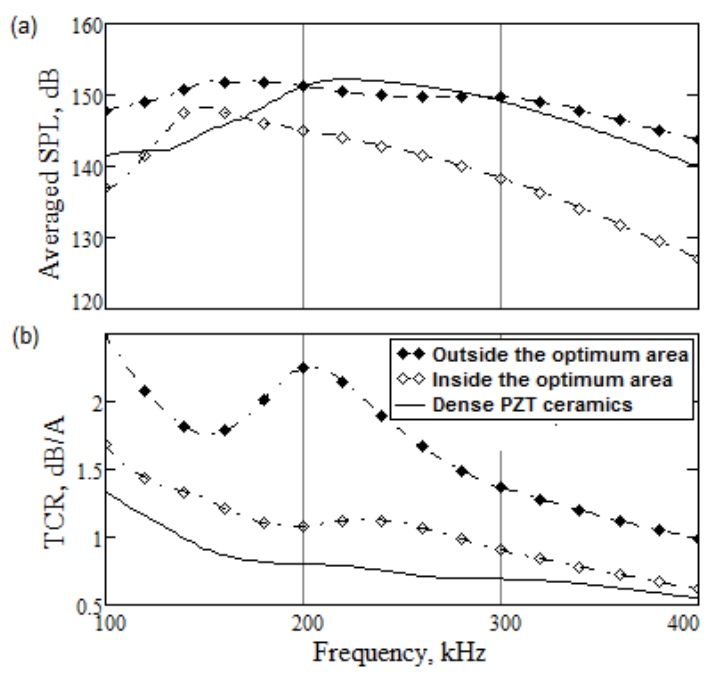

Figure 5. The frequency responses of SPL (a) and TCR (b).

successfully obtained for the porous piezocomposite materials of different connectivity in order to optimize the hydroacoustic performance of multilayered projector based on the active PZT layer with varied porosity. These effective modules were calculated using the $\mathrm{FE}$ method at the assumption of homogeneous and inhomogeneous polarization field. The last dependencies were used at the statement and solving the optimization problem due to the best agreement with the experimental data. Obtained dependencies allowed to reduce the number of design variables to six (porosity of an active layer; Young's modules of an acoustic window layer, protective and matching layers; mass and stiffness damping parameters of layers). On the base of the Pareto optimality the set of feasible designs in a six dimensional design space was reconstructed using three objectives: averaged sound pressure level, transmitting current response and the standard deviation of the SPL in a frequency range from 100 to $400 \mathrm{kHz}$. A comparative analysis of three examples of the simulated designs has been performed. It showed the best performance of a projector with porosity near $40 \%$ and elastic modules of intermediate layers tuned to achieve the best acoustic impedances matching between the structure and acoustic medium.

\section{Acknowledgements}

This work is partially supported by the Russian Foundation for the Basic Researches (Grant 12-08-31350) and by National Science Council of Taiwan (Project NSC992923-E-022-001-MY3).

\section{REFERENCES}

[1] R. Sathishkumar, "Micro Size Ultrasonic Transducer for
Marine Applications," Indian Journal of Science and Technology, Vol. 4, No. 1, 2010, pp. 8-13.

[2] I. Getman and S. Lopatin, "Theoretical and Experimental Investigation of the Porous PZT Ceramics," Ferroelectrics, Vol. 186, 1996, pp. 301-304. http://dx.doi.org/10.1080/00150199608218088

[3] R. Ramesh, H. Kara and C. R. Bowen, "Finite Element Modelling of Dense and Porous Piezoceramic Disc Hydrophones," Ultrasonics, Vol. 43, No. 3, 2005, pp. 173181. http://dx.doi.org/10.1016/j.ultras.2004.05.001

[4] A. N. Rybyanets, "Porous Piezoceramics: Theory, Technology, and Properties," IEEE Transactions on Ultrasonics, Ferroelectrics, and Frequency Control, Vol. 58, No. 7, 2011, pp. 1492-1507. http://dx.doi.org/10.1109/TUFFC.2011.1968

[5] V. Yu. Topolov and C. R. Bowen, "Electromechanical Properties in Composites Based on Ferroelectrics,” Springer, London, 2009.

[6] A. V. Nasedkin and M. S. Shevtsova, "Improved Finite Element Approaches for Modeling of Porous Piezocomposite Materials with Different Connectivity,” Ferroelectrics and Superconductors: Properties and Applications, Nova Science Publishers, Parinov, 2011, pp. 231-254.

[7] A. V. Nasedkin and M. S. Shevtsova, "Multiscale Computer Simulation of Piezoelectric Devices with Elements from Porous Piezoceramics," Physics and Mechanics of New Materials and Their Applications, Nova Science Publishers, Parinov, 2013, pp. 185-202.

[8] L. P. Khoroshun, B. P. Maslov and P. V. Leshchenko, "Prediction of Effective Properties of Piezoactive Composite Materials,” Kiev, Nauk Dumka, 1989.

[9] T. A. Witten and L. M. Sander, "Diffusion-Limited Aggregation: A Kinetic Critical Phenomenon,” Physical Review Letters, Vol. 47, No. 19, 1981, pp. 1400-1403. http://dx.doi.org/10.1103/PhysRevLett.47.1400

[10] R. Guo and C.-A. Wang, "Enhanced Piezoelectric Property of Porous Lead Zirconate Titanate Ceramics with One Dimensional Ordered Pore Structure," Journal of Applied Physics, No. 108, 2010, pp. 1-4.

[11] B. Jadidian and A. Winder, "Porous Piezoelectric Ceramics with 0 - 3 Connectivity," 2013. http://www.jwmed.com/docs.htm

[12] J. F. Li, K. Takagi, M. Ono, W. Pan and R. Watanabe, "Fabrication and Evaluation of Porous Piezoelectric Ceramics and Porosity-Graded Piezoelectric Actuators," Journal of the American Ceramic Society, Vol. 86, No. 7, 2003, pp. 1094-1098. shttp://dx.doi.org/10.1111/j.1151-2916.2003.tb03430.x

[13] A. N. Rybyanets and A. A. Rybyanets, "Ceramic Piezocomposites: Modeling, Technology, and Characterization," IEEE Transactions on Ultrasonics, Ferroelectrics, and Frequency Control, Vol. 58, No. 9, 2011, pp. 1757-1773. http://dx.doi.org/10.1109/TUFFC.2011.2013 